\title{
Im Schatten der DRG
}

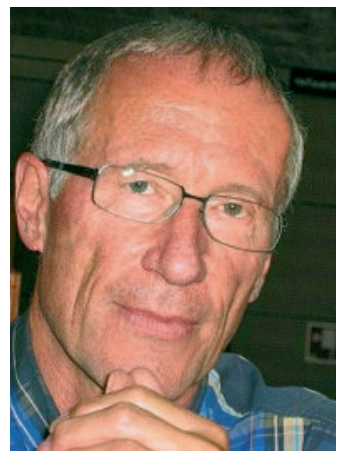

Erhard Taverna
Bis Ende 2011 sollen in der ganzen Schweiz die stationären Behandlungen mit Fallpauschalen (DRG) abgegolten werden. Der Kampf um den milliardenschweren Markt ist in vollem Gange, zum Beispiel, wenn es darum geht, die Kosten der Spitalprozesse von Medizinprodukten abzukoppeln. Die FMH, die bei Swiss DRG für einen hohen Eintrittspreis mitreden darf, teilt ihren Einfluss mit mächtigen Lobbyisten wie Kassen und Produzenten. Überaus aktiv ist in diesem Zusammenhang der Dachverband der Schweizerischen Handels- und Industrievereinigungen der Medizintechnik (FASMED). Da auch in Zeiten der Wirtschaftskrise rund 300 Schweizer Medizintechnik-Unternehmen jährlich um 5-10\% wachsen und circa 45000 Personen beschäftigen, sind sie bei der Neugestaltung der Spitalfinanzierung ein wichtiger Partner. Mit einer Exportrate von $66 \%$ sind sie überdies erfolgreich in einer Branche tätig, die international von teuren Firmenübernahmen geprägt wird. Der Medizintechnik wird eine goldene Zukunft vorausgesagt, ganz gleich, ob es sich um Infusionspumpen, orthopädische Implantate, beschichtete Stents oder Herzschrittmacher handelt. Noch herrschen Goldgräberzeiten, denn eine Markt-

\section{«Wo es um sehr viel Geld geht, und grosse Strukturveränderungen im Gange sind, wird nicht transparent verhandelt»}

ordnung wie in der Pharmabranche existiert nicht. Wochenaufenthalte für kardiologische Lernkurse in attraktiven Städten Europas oder exklusive Abkommen mit einzelnen Spitalverwaltungen sind durchaus üblich. Gemäss Statistik der Arbeitsgruppe Herzschrittmacher und Elektrophysiologie der SGK wurden 2007 hierzulande an 69 Zentren 3568 Schrittmacher erstimplantiert und weitere 1464 gewechselt. Die ICD-Implantationen haben sich von 2000-2007 verdreifacht. Bei Bruttomargen von bis 70\% für ein Gerät, das etwa so viel wie ein neuer VW Golf kostet, sind auch neue Impulse für die Aktienkurse der Hersteller garantiert. Ein ähnliches Bild zeigen die Katheterablationen, die sich im gleichen Zeitraum ebenfalls verdreifacht haben.

Deutschland macht vor, wie im Zeichen der DRG neue Investoren den ambulanten Markt entdecken. Seit 2004 dürfen Ärzte und Kliniken medizinische Versorgungszentren aufbauen, und Krankenkassen können seit 2007 zur Versorgung ihrer Versicherten Ver- träge mit Ärztenetzen oder mit neuen Betreibern abschliessen. Es zeichnet sich eine ähnliche Entwicklung wie in den USA und Grossbritannien ab, wo Supermarkt- und Drogerieketten Ambulanzen in ihren Filialen betreiben. Wal-Mart will bis 2010 rund 400 neue Praxen eröffnen, eine Entwicklung, die sich in Europa auch für Apotheken und Privatlabors abzeichnet. Auf der Suche nach neuen Anlagemöglichkeiten investieren selbst Schweizer Unternehmen in die eigens gegründete Private-Equity-Gesellschaft Deutscher Ärztefonds. Schwergewichte im Markt bleiben aber viele Krankenhäuser, wie die private Röhn-Klinik, ein Konzern, der systematisch Arztpraxen aufkauft und diese seinen ambulanten Versorgungszentren einverleibt.

Wo es um sehr viel Geld geht, und grosse Strukturveränderungen im Gange sind, wird nicht transparent verhandelt. Wer sich unter Konkurrenten eine gute Ausgangsposition sichern will, spielt mit verdeckten Karten, und wenn gar Spitäler um ihre Existenz bangen, ist es mit der Offenheit definitiv vorbei. Wie wäre es sonst zu erklären, dass ein erfahrener Kardiologe und langjährig ausgewiesener Fachmann für die Implantation von Schrittmachern im grössten Kanton von Spitälern ausgeschlossen wird? Selbst wenn ein Bezirksgericht ein Spital zur Zahlung von dessen Honorar-, Schadenersatz- und Genugtuungsansprüchen verpflichtet, lassen sich die Spitaldelegierten von der Gewinnsteigerung und der Kundenzufriedenheit blenden. Die neue Geschäftsstrategie setzt auf Expansion und Steigerung der Einnahmen im ambulanten und stationären Bereich. Kontrollinstanzen unterstützen einen Kurs, der die angestellten Chefs und leitenden Ärzte in Bonusempfänger gewinnorientierter öffentlicher Spitäler verwandelt. Für einen kostenbewussten Konsiliararzt scheint in diesem System kein Platz zu sein, denn eine Wirtschaftlichkeitsprüfung der eigenen Tätigkeit wird abgelehnt. Symptomatisch in diesem Fall sind auch die desinteressierten Reaktionen sogenannter Standesvertreter. Der amtierende FASMED-Präsident warnt vor Rationierung, Innovationsstau und Zweiklassenmedizin, sollten kostenintensive Produkte und Verfahren nicht von Fallpauschalen ausgenommen werden. Für seinen frommen Wunsch sind aber die Ärzte und ihre Vertreter zuständig: «Die therapeutische Freiheit des Arztes muss auch unter SwissDRG vollumfänglich gewährleistet sein. Der Arzt soll indikationsbezogen, individuell und mit dem Patienten die Wahl der geeigneten Therapie und den Einsatz der Medizintechnik treffen, die er verwenden will.» 